\title{
Los amargos beneficios del dulce. Azúcar, Cuba y deuda ecológica
}

\author{
Martín Rodrigo y Alharilla
}

Universitat Pompeu Fabra, Barcelona

\begin{abstract}
El artículo se situa en el paradigma de la historia ambiental. A partir de un enfoque a la vez microhistórico e inductivo, ofrece una reflexión sobre el vínculo existente entre la deforestación registrada en Cuba por efecto de la expansión del cultivo de la caña de azúcar y la transferencia de parte de las ganancias producidas por el azúcar a economías de otras latitudes, particularmente hacia Cataluña. Se analiza, en primer lugar, el proceso de implantación de las haciendas cañeras en la antigua provincia cubana de Las Villas, durante el segundo tercio del siglo XIX, y, a continuación, la trayectoria vital de varios hacendados de la región, los cuales acabaron instalándose en la etapa final de su vida en Cataluña, adonde trasladaron buena parte de los capitales acumulados en la isla. Por último, se apuntan diferentes evidencias que sugieren que el análisis planteado podría realizarse igualmente para otras regiones de Cuba así como para Puerto Rico.
\end{abstract}

Palabras Clave: Caña de azúcar, Cuba, Deforestación, Deuda ecológica

This article is placed within the paradigm of environmental history. From a focus that is both macrohistorical and inductive, it offers a reflection upon the link between the deforestation registered in Cuba as a result of the expansion of sugar cane cultivation, and the transfer of part of the gains produced by sugar towards economies in other latitudes, particularly towards Catalonia. The study analyzes first of all the establishment of sugar cane "haciendas" in the old Cuban province of Las Villas during the second third of the 19th century. It then inquires into the life trajectory of several "hacienda" owners of the region, who in the last period of their lives settled down in Catalonia, where they transferred a large part of the capital they had accumulated on the island. Finally, the essay points at evidence that suggests that the proposed analysis may be applied to other regions in Cuba as well as to Puerto Rico.

KEYWORDS: Sugar cane, Cuba, Ecological Debt.

La historia del azúcar en Cuba se ha convertido, probablemente, en el aspecto mejor estudiado de la historia económica insular. El carácter pionero de los trabajos de Roland T. Ely y de Manuel Moreno Fraginals ha sido completado, particularmente en los últimos diez años, con diversas 
monografías que, en su conjunto, ofrecen un panorama bastante completo en torno de la historia del cultivo, la transformación y la comercialización de la caña de azúcar y sus derivados en Cuba durante las tres últimas centurias. ${ }^{1}$ Para el siglo XVIII contamos con los estudios de Mercedes García mientras que las últimas décadas del siglo XIX (período ausente en $E l$ Ingenio, de Moreno Fraginals) han sido tratadas exhaustivamente por Fe Iglesias. ${ }^{2}$ Los trabajos de Allan Dye, Oscar Zanetti y Antonio Santamaría, por su parte, se han ocupado de analizar la industria azucarera cubana durante buena parte del siglo $\mathrm{XX} .^{3}$

Contamos, por otro lado, con diversos trabajos dedicados a analizar monográficamente determinadas realidades directamente vinculadas al sector azucarero cubano. A título de ejemplo, conocemos cómo funcionó el mercado esclavista en la isla. ${ }^{4}$ Sabemos, asimismo, que la red ferroviaria cubana nació en el siglo XIX impulsada por los hacendados del país ante la necesidad de reducir los costes de transporte y comercialización de los derivados de la caña. ${ }^{5}$ Conocemos también con precisión los intensos debates y programas generados ante la necesidad de aportar fuerza de trabajo para el sector, especialmente en los últimos años de vigencia de la esclavitud. ${ }^{6}$ Y, en el plano más social, los trabajos de Rebecca J. Scott, Ada Ferrer, Michael Zeuske y de otros autores han permitido analizar y describir de que forma tuvo lugar, en el campo y en los ingenios cubanos, el pro-

1 Ely, Roland T.: Cuando reinaba Su Majestad el Azúcar, La Habana, Imagen Contemporánea, 2001 [edición original de 1963]. Moreno Fraginals, Manuel: El Ingenio, La Habana, Editorial de Ciencias Sociales, 1974 [edición original de 1964].

2 García, Mercedes: La aventura de fundar ingenios. La refacción azucarera en la Habana del siglo XVIII, La Habana, Editorial de Ciencias Sociales, 2004. Iglesias, Fe: Del Ingenio al Central, La Habana, Editorial de Ciencias Sociales, 1999.

3 Dye, Allan: Cuban Sugar in the Age of Mass Production: Technology and the Economics of Cuban Sugar Central, New York, Stanford University Press, 1998. Zanetti, Oscar: Dinámica del estancamiento. El cambio tecnológico en la industria azucarera cubana, 1926-1958, La Habana, Instituto de Historia de Cuba, 1996; del mismo autor, Las manos en el dulce. Estado e intereses en la regulación de la industria azucarera cubana, 1926-1937, La Habana, Editorial de Ciencias Sociales, 2004. Santamaría, Antonio: Sin azúcar no hay país. La industria azucarera y la economía cubana (19191939), Sevilla, Escuela de Estudios Hispanoamericanos, 2002, así como “Azúcar y Revolución. El sector azucarero de la economía cubana en los doce primeros años de la revolución (1959-1970)", Revista de Historia Económica, 1994, XII/1, págs. 111-141.

4 Bergad, Laird W.; Iglesias. Fe; Barcia, M. Carmen: The Cuban Slave Market, 1790-1880, New York, Cambridge University Press, 1995.

5 Zanetti, Oscar; García, Alejandro: Caminos para el azúcar, La Habana, Editorial de Ciencias Sociales, 1987.

6 Balboa, Imilcy: Los brazos necesarios, Valencia, Fundación Instituto de Historia Social, 2000. 
ceso de transición de una mano de obra servil a una mano de obra jurídicamente libre. ${ }^{7}$

Ahora bien, no todas las aristas de la expansión azucarera han merecido idéntica atención. De hecho, una de las dimensiones hasta ahora menos tratadas de dicho proceso tiene que ver con sus implicaciones ecológicas. Sólo en tiempos recientes, los trabajos de Reinaldo Funes han venido a cubrir esta laguna. ${ }^{8}$ A medio camino entre la historia económica y la historia ambiental, este autor ha puesto de relieve cómo la imparable extensión del cultivo de la caña de azúcar en territorio cubano fue transformando el paisaje insular, de forma notable e irreversible. Reinaldo Funes ha insistido, particularmente, en el carácter deforestador de dicha expansión. Según sus propias palabras, si bien el azúcar no fue "el único factor que propició la desaparición de la cubierta forestal existente [en Cuba] a la llegada de los europeos ... por sus características, la economía azucarera se convirtió ... en la principal causa de deforestación" de la isla. ${ }^{9}$ Tomando como punto de partida, precisamente, dicha realidad, este trabajo insiste en la utilidad de incorporar la dimensión ambiental al análisis histórico. Y pretende, particularmente, aplicar al caso cubano un concepto de factura relativamente reciente: la noción de deuda ecológica o pasivo ambiental. Pero... ¿qué es la deuda ecológica?

En 1990, el Instituto de Ecología Política de Chile publicó un documento que ponía de relieve los efectos para el conjunto del planeta (también para los países del Sur) de la emisión de gases clorofluorocarbonados (CFCs) por empresas del Norte, sugiriendo por tanto que se producía así

7 Scott, Rebecca J.: Slave Emancipation in Cuba. The Transition to Free Labor, 1860-1899, Princeton, Princeton University Press, 1988 [existe traducción castellana, México, Fondo de Cultura Económica, 1989]. Ferrer, Ada: Insurgent Cuba. Race, Nation and Revolution, 1868-1898, The University of North Carolina Press, Chapel Hill, 1999. Zeuske, Michael: "'Los negros hicimos la independencia': aspectos de la movilización afrocubana en un hinterland cubano. Cienfuegos entre colonia y República", en Martínez, Fernando et alii: Espacios, silencios y los sentidos de la libertad. Cuba entre 1878 y 1912, La Habana, Ediciones La Unión, 2001. A destacar asimismo varios de los trabajos incluidos en el volúmen, Piqueras, José A. (comp): Azúcar y esclavitud en el final del trabajo forzado, Madrid, Fondo de Cultura Económica, 2002.

8 Los recientes trabajos de Reinaldo Funes están cubriendo ese vacío. Aportaciones como: "Azúcar y deforestación: una aproximación a la historia ambiental en Cuba", en González de Molina, Manuel; Martínez Alier, Joan (eds): Naturaleza transformada, Barcelona, Icaria, 2001, págs. 183-209. Del mismo autor: "Tierras cansadas y quemadores de bagazo verde. La interacción con el medio natural y los cambios en la industria azucarera cubana desde mediados del siglo XIX”, en Piqueras, José A. (comp): Azúcar y esclavitud ..., págs. 186-213; y sobre todo su libro: De bosque a sabana. Azúcar, deforestación y medio ambiente en Cuba (1492-926), México, Siglo XXI, 2004.

9 Funes, R: “Azúcar y deforestación ...”, pág. 194. 
una deuda ecológica del Norte con el Sur. En posteriores encuentros internacionales, como en las reuniones alternativas a la Cumbre de Río, de 1992, dicha noción acabó de tomar forma, amplió su significado original y pasó a ser utilizada no sólo por colectivos ciudadanos sino también por investigadores de disciplinas diversas. ${ }^{10}$ Desde entonces la noción de deuda ecológica, denominada también pasivo ambiental, ha estado presente en diversos estudios de diferente carácter (económicos, históricos, jurídicos). ${ }^{11}$ Si bien las acepciones más difundidas del significado de la deuda ecológica tienen que ver tanto con la contaminación ambiental como (especialmente en América Latina) con el expolio de los recursos naturales lo cierto es que su significado no se agota únicamente en esos términos. ${ }^{12} \mathrm{Se}$ la ha definido, de forma más general, como "la responsabilidad que tienen los países industrializados por la destrucción paulatina del planeta como efecto de sus formas de producción y consumo"; y a pesar de que el uso de la noción de pasivo ambiental está muy vinculada a realidades actuales, se entiende que "la Deuda Ecológica se genera en la época colonial y se sigue incrementando hasta nuestros días" a través de diferentes instrumentos, entre los cuales cabe incluir "el uso y degradación de las mejores tierras ... para establecer cultivos de exportación". ${ }^{13}$

En la medida que el auge azucarero cubano se explica por la posibilidad de colocar el dulce en mercados externos a la isla, parece claro que la expansión del cultivo de la caña para su exportación y la de sus derivados pudo generar en Cuba un pasivo ambiental no negligible aunque difícil de cuantificar. ${ }^{14}$ Entiendo que al menos dos de los supuestos de la deuda ecológica son útiles para nuestro campo de estudio: por un lado, en buena parte del país se sustituyó la diversidad biológica por el monocultivo azucare-

10 Prefacio de Martínez Alier, Joan a: Colectivo de Difusión de la Deuda Ecológica: Deuda Ecológica. El Norte está en deuda con los países del Sur, Editado por el Observatorio de la Deuda en la Globalización, 2002.

11 Para una versión de sus implicaciones jurídicas Borrero Navia, José M.: Los derechos ambientales. Una visión desde el Sur, Cali, FIPMA/CELA, 1994. Una visión de la potencialidad explicativa de la deuda ecológica frente a las explicaciones financieras más al uso de las relaciones NorteSur en: Martínez Alier, Joan; Oliveres, Arcadi: ¿Quién debe a quién? Deuda Ecológica y Deuda Externa, Barcelona, Icaria, 2002. Una definición harto compleja del concepto en Dillon, John: "Deuda ecológica. El Sur dice al Norte 'Es hora de pagar'”, Ecología Política, n. ${ }^{\circ} 20$, págs. 131-151.

12 Un precedente avant la lettre de la deuda ecológica de Europa con la América hispana en Galeano, Eduardo: Las venas abiertas de América latina, México DF, Siglo XXI, 1975.

13 Extraído de Acción Ecológica, 1999.

14 Sobre la dificultad y necesidad de cuantificar la deuda ecológica, Martínez Alier, Joan: "Ecological debt - external debt", Quito, Acción Ecológica, 1998. 
ro; así como, por otro lado, que parte de los beneficios de dicho proceso emigraron fuera de la isla. Partiendo de ambos hechos las páginas que siguen intentarán ofrecer una reflexión sobre el vínculo entre la deforestación registrada en Cuba por efecto de la expansión cañera y la transferencia de parte de las ganancias producidas por el azúcar a economías de otras latitudes, particularmente hacia Cataluña. ${ }^{15}$ Una reflexión que se ubica cronológicamente en el segundo y el tercer tercio del siglo XIX.

El trabajo se inicia con un breve apartado dedicado a ofrecer algunos datos sobre el proceso deforestador registrado en la primera mitad del siglo XIX en las regiones occidental y central de la isla. Intentaré ofrecer, a continuación, un análisis centrado en la antigua provincia de Las Villas. Para éllo situaré, en primer lugar y de manera genérica, el proceso de extensión de las plantaciones de caña en las jurisdicciones de Sagua la Grande, de Cienfuegos y de Remedios. En segundo lugar, describiré someramente la trayectoria vital de varios hacendados instalados en dicha región. Hombres de negocio que compartieron un mismo denominador: tras enriquecerse en las décadas centrales del siglo XIX, en la zona central de Cuba, todos éllos acabaron instalándose en la etapa final de su vida en Cataluña, adonde trasladaron buena parte de los capitales acumulados en la isla. Se trata, pues, de utilizar un enfoque a la vez microhistórico e inductivo. Describiré la trayectoria vital de unos hombres de negocio que fueron, como se verá, no sólo partícipes sino verdaderos impulsores del boom azucarero registrado en los hinterlands de Cienfuegos, Sagua la Grande y Remedios, a mediados del siglo XIX. Y apuntaré, por último, diferentes evidencias que sugieren que este tipo de análisis y de aproximación podría realizarse para otras regiones de Cuba así como también para la isla de Puerto Rico.

\section{Azúcar y deforestación en Cuba}

En 1828 José Antonio Saco publicó una extensa nota titulada significativamente Montes o bosques en la isla de Cuba. Saco partía de una memoria presentada treinta y dos años antes en la Sociedad Patriótica de La Habana en la que su autor, el hacendado José Ricardo O'Farril, lamen-

15 Aunque existen monografías (como la de Zanetti, Oscar; García, Alejandro: United Fruit Company: un caso de dominio imperialista en Cuba, La Habana, Editorial de Ciencias Sociales, 1976) que se han preocupado de estudiar el impacto del capital extranjero en la economía cubana, no podemos decir que abunden los estudios sobre la fuga de capitales fuera de la isla. 
taba la destrucción que a finales del siglo XVIII estaban sufriendo los montes del entorno capitalino vaticinando que "si no se tomaban medidas oportunas para contener el mal que amezazaba [a la capital], La Habana, dentro de 30 años, vería alejar sus montes hasta la distancia de 40 leguas". En $1828 \mathrm{Saco}$, no sólo podía confirmar que "una experiencia dolorosa ha venido a cumplir tan triste vaticinio" sino que alertaba que "aún en algunas partes de las menos pobladas y cultivadas de la Isla, ya se empieza [a notar] la falta de bosques". ${ }^{16}$ Curiosamente, en esos mismos años uno de los adversarios intelectuales de Saco, Ramón de la Sagra, tuvo idéntica preocupación que su oponente al lamentar las tumbas de los montes causadas por la voracidad del despliegue azucarero: "volviendo la vista de los bosques destruidos a los campos cultivados", Sagra deploraba los efectos de las hachas exterminadoras y de las talas asoladoras que transformaban "en llanuras estériles y abrasadas, terrenos antes pingües y frondosos" concluyendo que "donde se aniquilen los árboles, una escena de soledad y muerte sustituirá al risueño espectáculo de una naturaleza jóven y agreste". ${ }^{17}$

El propio Moreno Fraginals en su monumental obra El Ingenio dedicó un breve apartado a La muerte del bosque. Según sus cálculos "a fines del siglo XVIII se tumbaban anualmente 500 caballerías de bosque $(6.710$ hectáreas) para quemarlo como leña en los ingenios. Y otras muchas más para el fomento de las nuevas fábricas"; mientras que "en 1819 la cifra promedio subió a 1000 [caballerías] (13.420 ha)". Moreno recoge asimismo los cálculos de dos hacendados quienes, en 1830, cifraban en 2.000 caballerías (es decir, 26.840 hectáreas) las tierras desmontadas, para culminar diciendo que en 1844 esa cifra se calculaba en el doble, 53.680 hectáreas. Tal como concluye el historiador cubano, "el derecho a desforestar la isla fue una de las grandes victorias legales de la sacarocracia". ${ }^{18}$ Extremo confirmado por Reinaldo Funes en un trabajo que analiza la incipiente victoria de los hacendados habaneros frente a las autoridades de Marina por el disfrute de las maderas. ${ }^{19}$

16 Saco, José Antonio: Obras, La Habana, Imagen Contemporánea, 2001, vol I, págs. 155-160.

17 De la Sagra, Ramón: Historia económico-política y estadística de la isla de Cuba, Habana, 1831, pág. 84 .

18 Moreno, M.: El Ingenio ..., vol. I, págs. 157-163. A Roland T. Ely, sin embargo, no parecieron preocuparle las implicaciones deforestadoras de la expansión cañera pues en su libro clásico apenas le dedicó alguna reflexión muy marginal, Ely, R. T.: Cuando reinaba ....

19 Funes, Reinaldo: "Los conflictos por el acceso a la madera en La Habana: hacendados vs. Marina (1774-1815)", en Piqueras, José A. (ed): Diez nuevas miradas de Historia de Cuba, Castelló, Publicacions de la Universitat Jaume I, págs. 67-90. 
Diferentes estudios de alcance regional han podido analizar que lo descrito por Saco, Sagra y Moreno para el entorno habanero se acabó reproduciendo en buena parte de la geografía insular. De hecho, "hasta fines del siglo XVIII fue la deforestación un fenómeno de grandes proporciones sólo en los territorios de la actual provincia de La Habana. Durante el siglo XIX se extendió hacia el occidente y centro de la isla [y] por último, en el primer cuarto del siglo XX, irrumpió en una magnitud sin precedentes hacia la zona oriental del país". ${ }^{20}$ No en vano, tras cubrir buena parte de la llanura habanera, la frontera del azúcar (por usar el término empleado por Laird W. Bergad) se expandió hacia el este, transformando radicalmente la región de Matanzas a lo largo del primer tercio del siglo XIX. ${ }^{21}$ Más allá de Matanzas, el azúcar siguió avanzando hacia el este cubriendo la antigua provincia de Las Villas en un proceso que se aceleró enormemente durante el segundo tercio del siglo XIX. Análisis regionales centrados en las jurisdicciones de Cienfuegos, de Sagua la Grande, de Trinidad o de Remedios, así lo confirman..$^{22}$ En las últimas décadas de la centuria, el azúcar siguió conquistando territorios en la isla, transformando buena parte del paisaje de la región centro-oriental y oriental de Cuba. Un proceso en el que la colonización del azúcar se produjo paralelamente a la profunda transformación de las unidades de producción de la caña que dio pie a los modernos centrales. ${ }^{23}$

\section{Capitales y capitalistas, de las villas a Barcelona}

Sagua la Grande fue fundada en 1812 por el veguero Domingo Caballero si bien cinco años después "no había allí más edificios que la casita de vivienda de [la] modesta finca [del fundador] y otras dos de dos isleños

20 Funes, R: “Azúcar y deforestación ...”, pág. 203.

21 Bergad, Laird W.: Cuban Rural Society in the Nineteenth Century. The Social and Economic History of Monoculture in Matanzas, Princeton, Princeton University Press, 1990.

22 García, Orlando: "Estudio de la economía cienfueguera desde la fundación de la colonia Fernandina de Jagua hasta mediados del siglo XIX”, Islas, núm. 55-56, sept. 1976-abril 1977, págs. 117-169. Venegas, Hernán: "Consideraciones en torno a la economía remediana colonial”, Islas, núm. 67, 1980, págs. 11-79; "Acerca del proceso de concentración y centralización de la industria azucarera en la región remediana a fines del siglo XIX”, Islas, núm. 73, 1982, págs. 63-121. Guerra, Carmen: “Acerca de la relación azúcar-esclavitud en la región cienfueguera", Islas, núm. 89, 1988, págs. 26-40; Guerra, Carmen; Jiménez, Isabel: "La industria azucarera cienfueguera en el siglo XIX: Notas históricas para su estudio", Islas, núm. 91, 1988, págs. 42-76; García, Gloria: "Crecimiento económico, azúcar y campesinado en Remedios (1846-1865), Islas, 1993, págs. 100-106.

23 F. Iglesias: Del Ingenio .... 
de Canarias atraídos por Caballero". Solamente en 1822 pudo Sagua dotarse de su primer Ayuntamiento. ${ }^{24}$ Por su parte, la colonia Fernandina de Jagua (precedente de la actual Cienfuegos) fue fundada en 1819. Ocho años después, en 1827, consiguió que su puerto se habilitase para el comercio, facilitando así la posterior extensión azucarera de la comarca. Una descripción de entonces dice que en 1827, en Cienfuegos, "casi todo está aun en proyecto, pues en el día sólo aparecen algunos grupos informes de edificios desiguales, varios de vistosa construcción y la mayor parte pequeños aislados y de poca consistencia". La ciudad tenía entonces no más de 841 habitantes mientras que se cifraban en 714 los residentes en los campos cercanos. Apenas había en sus inmediaciones seis haciendas azúcareras: "4 ingenios regulares con sus alambiques y tejares que no son de la jurisdicción de la colonia y dos más de los últimos, pertenecientes a los colonos", donde laboraban un total de 175 esclavos. La misma fuente describe Sagua la Grande, también en 1827, como un "pueblo naciente y pequeño puerto a la margen izquierda del río del mismo nombre" con "un mal cuartel para destacamento de infantería y caballería", una única tienda de ropa, sólo dos panaderías, apenas 463 habitantes (de los que 71 eran esclavos) "y en sus inmediaciones dos pequeños ingenios, varios potreros [y] vegas". En los otros núcleos de población cercanos, la población esclava era relativamente reducida: 27 esclavos en Quemado de Güines (sobre un total de 183 habitantes) y 24 en San Narciso de Álvarez (sobre una población de 260 individuos). Únicamente en la pequeña colonia de Santo Domingo parece apreciarse una cierta presencia de esclavos: 140 esclavos para 872 almas. No obstante, ni en Álvarez, ni en Santo Domingo ni en Quemado de Güines aparecía entonces noticia alguna de fincas azucareras. ${ }^{25}$

Al parecer, el reparto de las primeras haciendas a orillas del río Sagua tuvo lugar en 1835, fundándose en ese año cuatro ingenios que "dieron a conocer la bondad de los terrenos atrayendo colonos respetables con lo que tomó vida la agricultura y principio el comercio del pueblo" de Sagua la Grande. ${ }^{26} \mathrm{El}$ impulso del azúcar a orillas del río Sagua fue tan rápido como espectacular. En los primeros años 1840 el doctor J. G. F. Wudermann remontó el curso del Sagua, pudiendo entonces ver y describir "las ricas

24 Jacobo de la Pezuela: Diccionario geográfico, estadístico, histórico de la isla de Cuba, Madrid, 1863, vol. IV, pág. 387.

25 Cuadro estadístico de la siempre fiel isla de Cuba correspondiente al año de 1827, La Habana, 1829, págs. 75-79.

26 De la Pezuela, J.: Diccionario geográfico ..., vol. IV, pág. 376. 
tierras negras elevadas sobre el río y espesamente sembradas de caña de azúcar. Hasta más de una milla de la costa no se veían árboles. Los límites de cada cañaveral estaban señalados sólo por los extensos almacenes e instalaciones de los respectivos ingenios. Las máquinas de vapor estaban todas en funcionamiento, exhalando columnas de humo por sus altas chimeneas, y con las casas de los hacendados que estaban junto a las márgenes del río daban al paisaje un tono muy animado". ${ }^{27}$ De hecho, la población de Sagua la Grande y de los partidos vecinos había aumentado de forma igualmente rápida y espectacular: en 1841 habitaban el partido de Sagua un total de 5.846 personas, de los que el 37'9 por 100 eran esclavos. $\mathrm{Y}$ es que en los catorce años anteriores la cifra de población esclava de Sagua la Grande se había multiplicando por 31 (pasando de 71 esclavos negros en 1827 a 2.214 en 1841). En su conjunto, en lo que luego sería la jurisdicción de Sagua la Grande, se contabilizaban en 184119.526 habitantes, de los que 2.513 vivían en los 61 ingenios de la zona y 15.410 en el resto de fincas rurales de la región. ${ }^{28} \mathrm{El}$ azúcar fue el responsable último tanto del empuje productivo y demográfico de la zona como de la consiguiente deforestación: en 1862 se decía que más de la mitad de la extensión del partido de Sagua la Grande "continua [todavía] cubierta de bosques de todas clases que se desmontan a medida que van estendiendo (sic) sus plantíos de caña los ricos ingenios que ocupan las orillas" del río Sagua. ${ }^{29}$

La expansión de Cienfuegos no fue menos espectacular que la de su vecina Sagua: de los escasos 1.600 habitantes de 1.827 se había pasado a un total de 5.279 catorce años después, de los que el 36,5 por 100 eran esclavos. ${ }^{30}$ Una expansión igualmente vinculada al azúcar: según recogía un cronista cienfueguero de origen catalan, Enrique Edo Llop, de los 5 ingenios y 5 trapiches que allí había en 1830 se pasó a 26 ingenios en 1839 . Sabemos por otro lado que en 1846 la región de Cienfuegos contaba ya con 71 ingenios y que en 1856 dicho número había aumentado hasta $112 .{ }^{31}$ En el marco de la segunda visita que Ramón de la Sagra efectuó a Cuba, realizó una excursión "en el invierno de 1859 a 1860 por algunas riquísimas y feraces comarcas" cercanas a Cienfuegos, pudiendo confirmar "los hechos

27 Wudermann, J. G. F.: Notes on Cuba, Boston, 1844; recogido y traducido por Ely, R. T.: Cuando reinaba ..., pág. 363.

28 Resumen del censo de población de la isla de Cuba a fin del año de 1841, La Habana, 1842, págs. $42-43$.

29 De la Pezuela, J.: Diccionario geográfico ..., vol. IV, pág. 382 [El subrayado es mío].

30 Resumen del censo ..., págs. 44-45.

31 García, O.: "Estudio de la economía ...”, págs. 137-138. 
que de muy antiguo [decía el gallego] venimos deplorando", es decir, como "las talas salvajes ... del aspecto seductor de ricos jardines pasaron a ofrecer tristes horizontes desnudos y solitarios". ${ }^{32} \mathrm{El}$ mismo proceso se estaba produciendo en Remedios. Hernán Venegas ha señalado "lo tardío del desarrollo azucarero remediano ... [un desarrollo que] no alcanzó a la antigua jurisdicción colonial remediana hasta mediados del siglo XIX" ${ }^{33}$ La superficie dedicada en Remedios al cultivo del azúcar pasó de 4.650 hectáreas en 1855 a 7.718 hectáreas diez años después, lo que supuso que para 1865 casi la mitad de las tierras cultivadas se dedicasen a la caña. De hecho, en Remedios, la producción de azúcar por habitante pasó de 134 kilos en 1846 a 320 en $1865 .{ }^{34}$

Parte del auge azucarero tanto de Cienfuegos como de Sagua la Grande o de Remedios se ha explicado por la notable presencia de capital foráneo en ambas comarcas. Orlando García ha señalado la labor de hacendados trinitarios y habaneros en el despegue cañero de Cienfuegos mientras que Roland T. Ely, por su parte, ha puesto de relieve igualmente como diferentes hacendados de La Habana, Matanzas o Cárdenas (tales que Ann Phinney, James Macomb o la família Drake Del Castillo) fundaron ingenios en Sagua la Grande. ${ }^{35}$ No es menos cierto, sin embargo, que junto a habaneros, matanceros o trinitarios hubo también otros hombres de negocio que se incorporaron ex novo en los intersticios de las economías azucareras de Sagua la Grande, Cienfuegos, Remedios (e incluso de Santa Clara) de cuya expansión fueron no sólo partícipes sino además destacados protagonistas. Nos vamos a ocupar, sobre todo, de siete de éllos. Siete hacendados diferentes los cuales fomentaron y cultivaron un total de once ingenios diferentes: los hermanos Pablo Luis y Tomás Ribalta, Agustín Goytisolo, Francisco de Sola, José P. Taltavull, Joaquín Fábregas y José Carbó.

Los hermanos José, Pablo Luis y Tomás Ribalta Serra abandonaron su Barcelona natal para intentar hacer fortuna en Cuba. El primero no lo consiguió pues falleció en Sagua la Grande a los pocos años de su arribada a la isla. Sus dos hermanos, sin embargo, fueron capaces de fomentar hasta

32 De la Sagra, Ramón: Cuba en 1860 o sea cuadro de sus adelantos en la población, la agricultura, el comercio y las rentas públicas, París, 1862, pág. 53.

33 Venegas, Hernán: "Acerca del proceso de concentración y centralización de la industria azucarera en la región remediana a fines del siglo XIX”, Islas, núm. 73, sept-dic. 1982, pág. 63-121; del mismo autor puede consultarse también: "Consideraciones en torno a la economía remediana colonial", Islas, núm. 67, sept-dic. 1980, págs. 11-79.

34 García, G.: "Crecimiento económico ...”.

35 García, O. "Estudio de la economía...”. Ely, R. T.: Cuando reinaba ... 
cuatro ingenios diferentes en la gran Antilla. Desde su residencia en Santa Clara, Pablo Luis Ribalta impulsó en los años 1840 una hacienda cañera en la jurisdicción de Cienfuegos: el ingenio Santa Marta, ubicado en el partido de Las Casas, el cual contaba, en 1873, con una dotación de 116 esclavos, 16 colonos chinos y 6 libertos bajo patronato. El segundo ingenio fomentado por Pablo Luis Ribalta tomó el nombre de Rosa (en homenaje a su primera esposa, Rosa Mora) y se ubicó junto al ingenio Santa Teresa, propiedad como veremos de su hermano Tomás, aunque la división administrativa quiso que el segundo perteneciese a la jurisdicción de Sagua la Grande mientras que el ingenio Rosa, de Pablo Luis Ribalta, dependía del partido de Santa Clara. Una finca que contaba, en 1873, con 158 esclavos (de los que veinte habían huido), 10 colonos asiáticos (incluyendo cuatro huidos) y 10 libertos bajo patronato. ${ }^{36}$

El ingenio Santa Teresa se ubicó en el partido de Amaro, jurisdicción de Sagua la Grande. Una finca que acabó sumando un total de 63 caballerías en la que nació, en diciembre de 1868, Esteban Santa Teresa, quien tomó después el nombre de Esteban Montejo. Un interesante personaje cuya centenaria vida inspiró al novelista y etnólogo cubano Miguel Barnet en la elaboración de su conocido libro Biografía de un cimarrón; novela en la que aportó, no obstante, notables dosis de ficción, como ha demostrado Michael Zeuske. ${ }^{37}$ Por otro lado, la subasta del ingenio El Consejo, cercano al Santa Teresa, permitió a Tomás Ribalta hacerse con una nueva finca, a la que bautizó Santo Tomás, y cuya posesión tomó en noviembre de $1862 .{ }^{38} \mathrm{Su}$ ascendente sobre el municipio fue tal que Tomás Ribalta ejerció dos veces como alcalde de Sagua la Grande, la primera en 1860.

También en Sagua la Grande se instaló el medico catalán Joaquín Fábregas Estrada. Nacido en Piera, en 1820, Fábregas no se limitó en Cuba a ejercer su profesión sino que se integró plenamente en la vida pública de su ciudad adoptiva, ejerciendo como regidor del municipio y, especialmente, como Síndico Procurador General del mismo. ${ }^{39}$ Arribado a Sagua la

36 Los datos de la dotación de los ingenios Santa Marta y Rosa en: AHPVC, Registro de la Propiedad de Santa Clara, tomo III, fols. 76-90.

37 Zeuske, Michael: "El 'Cimarrón' y las consecuencias de la guerra del 95. Un repaso de la biografía de Esteban Montejo", Revista de Indias, vol. 212, 1998, págs. 65-84; completado con "Novedades de Esteban Montejo", Revista de Indias, vol. 216, 1999, págs. 521-525.

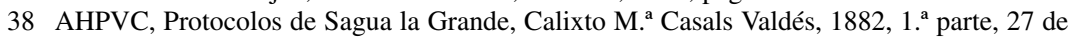
marzo de 1882, fols. 503-555; 1888, 1 . $^{\text {a }}$ parte, 26 de enero de 1888, fols. 104-180.

39 El Sagua, año XVIII, núm. 90, 11 de noviembre de 1869, pág. 2; núm. 101, 19 de diciembre de 1869 , pág. 1 . 
Grande en medio del auge azucarero que estaba transformando la jurisdicción, Joaquín Fábregas se interesó por el negocio del dulce, asociándose, por ejemplo, con el matrimonio formado por Pedro Augusto Aveilhé y María Moliné con quienes llegó a compartir la propiedad del ingenio Delta, cuya mitad compró Fábregas en febrero de 1865 tras pagar 105.000 pesos fuertes. Contentos con su proceder, los consortes Aveilhé-Moliné aconsejaban a sus herederos que "trat[asen] de continuar en esa sociedad a fin de que siempre se conserve en buen estado la finca ... cuyo encargo les hacemos atendiendo a la mucha confianza que tenemos con el citado [Joaquín] Fábregas". Por cierto que en dicho testamento ambos cónyugues afirmaban que los beneficios netos del ingenio Delta en su última zafra habían sido exactamente de veinte mil pesos, lo que nos da una idea de cual era el volumen de ganancias acumuladas por hacendados como el propio Fábregas. Años después, Joaquín Fabregas compró el ingenio San Joaquín, ubicado en el partido de San Diego del Valle, jurisdicción de Santa Clara. Además, en la medida que Fábregas prestaba dinero a otros hacendados, se beneficiaba de las ganancias producidas por otras fincas azucareras, como el ingenio Santa Ana, en Sagua la Grande, propiedad del norteamericano Jorge Knight. ${ }^{40}$

No en Sagua la Grande sino en Cienfuegos se instaló el vasco Agustín Goytisolo Lezarzaburu, el cual llegaría a amasar una gran fortuna merced al cultivo de la caña. Instalado en Cuba, en la década de 1830, Goytisolo llegó a fomentar hasta tres ingenios diferentes. En unión de su cuñado Antonio Arce, fomentó primero la hacienda Simpatía, en los años 1840. En marzo de 1850 compró, de su propio peculio, una segunda finca a la que bautizó Lequeitio, en recuerdo de su villa natal. Y, posteriormente, compró al trinitario Conde de Casa Brunet (quien había trasladado su residencia a Europa a finales de los años 1860) un tercer ingenio, al que bautizó San Agustín, en el término municipal de Santa Isabel de las Lajas. ${ }^{41}$ Como Tomás Ribalta en Sagua la Grande, también Agustín Goytisolo llegaría a ejercer, en 1871, como alcalde de su ciudad, en este caso, de Cienfuegos.

40 AHPVC, Protocolos de Sagua la Grande, Ramón de Iglesias, 1865, 1. ${ }^{\text {a }}$ parte, 28 de julio de

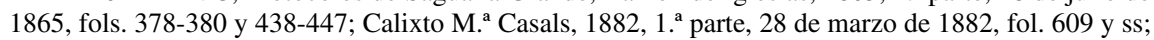
1883, 3. ${ }^{\mathrm{a}}$ parte, 3 de septiembre de 1883, fols. 1070-1072.

41 Rodrigo, Martín: "Los ingenios San Agustín y Lequeitio (Cienfuegos): un estudio de caso sobre la rentabilidad del negocio del azúcar en la transición de la esclavitud al trabajo asalariado (18701886)", en Piqueras, José A. (comp): Azúcar y esclavitud ...., págs. 252-268. 
Allí coincidió tanto con el menorquín José Pedro Taltavull García como con el vasco Francisco de Sola Nanclares. Abogado de formación, Francisco de Sola ejerció su profesión en Cienfuegos. Al constituirse, en julio de 1841, el primer Colegio de Abogados de Cienfuegos y Santa Clara, Sola ostentó el cargo de Segundo Diputado del mismo. En Cienfuegos pudo asimismo ahorrar el capital suficiente con el que comprar, en diciembre de 1845, un ingenio en el partido de Yaguaramas (cercano a la finca Simpatía, de los Goytisolo) al que denominó Cieneguita y que llegaría a sumar más de 1.300 hectáreas de terreno. ${ }^{42}$ Francisco de Sola llegaría además a desarrollar una intensa vida pública en la isla. Alcalde ordinario de Cienfuegos en diferentes años, como en 1859 y en 1864, Sola actuó como portavoz y como candidato frustrado de los sectores más integristas de Cienfuegos en las elecciones a la Junta de Información de $1866 .{ }^{43}$

José P. Taltavull, por su parte, había emigrado a Cienfuegos en 1839, con veintidós años. Se asoció con su hermano uterino Pedro Antonio Font García en la firma comercial Font y Cía, empresa que fomentó el ingenio Caridad, ubicado en el partido de Las Casas, es decir, cercano al ingenio Santa Marta, de Pablo Luis Ribalta. También el catalán José Carbó Martinell, natural de Sant Feliu de Guíxols, quiso dar el salto de la actividad mercantil al fomento y cultivo de haciendas cañeras. Instalado en la ciudad de Matanzas, José Carbó no pudo, sin embargo, fomentar ningun ingenio en el entorno matancero pues, a mediados del siglo XIX, no había allí terrenos vírgenes para desmontar. Debió centrar entonces sus esfuerzos en la jurisdicción de Remedios, concretamente en el partido de Yaguajay, es decir, en la zona más oriental de la región de Las Villas. Allí Carbó fundó y fomentó el ingenio Santa Catalina, cuya propiedad acreditaba en 1859, si no antes. A la altura de 1877 el ingenio Santa Catalina sumaba 44 caballerías de terreno (de las que se cultivaban 26) y contaba con una dotación de 89 esclavos, 19 chinos y 21 trabajadores asalariados. ${ }^{44}$ Cabe señalar que en los terrenos lindantes al Santa Catalina sendos catalanes fundaron otros dos ingenios, me refiero a José Bofill (propietario del Océano) y a Juan Font (de la finca Belencita).

42 AHPC, Escribanía de J. J. Verdaguer, Manual de 1885, 3 de julio de 1885, fols. 1263-1269.

43 Edo, Enrique: Memoria histórica de Cienfuegos y su jurisdicción, La Habana, 1943 [3. a edición], págs. 67, 176, 224 y 268.

44 Dirección General de Hacienda: Noticias de los ingenios y fincas azucareras que en estado de producción existen actualmente en toda la isla, La Habana, 1877. 


\section{CUADRO 1}

\section{ALGUNOS INGENIOS FOMENTADOS EN LA REGIÓN DE LAS VILLAS POR INDIANOS AVECINDADOS EN CATALUÑA}

\begin{tabular}{llll} 
Propietario & Ingenio & Partido & Jurisdicción \\
\hline Agustín Goytisolo Lezarzaburu & Simpatía & Yaguaramas & Cienfuegos \\
& $\begin{array}{l}\text { Lequeitio } \\
\text { San Agustín }\end{array}$ & $\begin{array}{l}\text { Cartagena } \\
\text { Lajas }\end{array}$ & Cienfuegos \\
Cienfuegos \\
José P. Taltavull García & Caridad & Las Casas & Cienfuegos \\
Pablo Luis Ribalta Serra & Santa Marta & Las Casas & Cienfuegos \\
& Rosa & Las Niguas & Santa Clara \\
Tomás Ribalta Serra & Santa Teresa & Amaro & Sagua la Grande \\
& Santo Tomás & Amaro & Sagua la Grande \\
Francisco de Sola Nanclares & Cieneguita & Yaguaramas & Cienfuegos \\
Joaquín Fábregas Estrada & San Joaquín & S. Diego del Valle & Santa Clara \\
José Carbó Martinell & Santa Catalina & Yaguajay & Remedios \\
\hline
\end{tabular}

Fuente: véase texto.

De los siete personajes analizados, seis se instalaron en Cataluña en la fase final de sus vidas. Sólo Pablo Luis Ribalta no puedo regresar a su Barcelona natal al fallecer prematuramente en Santa Clara, en junio de 1871. En esa fecha, su hermano Tomás Ribalta residía ya en la capital catalana. Nombrado su único y universal heredero Pablo Luis Ribalta quiso transferir post-mortem parte de su fortuna a su hermano Tomás, quién de esta manera incrementó notablemente su patrimonio. En concreto, Tomás Ribalta heredó de su hermano no menos de 1.286 .517 pesetas de la época quedando asimismo encargado de la administración y el usufructo de los bienes que Pablo Luis Ribalta había asignado a su impúber hija adoptiva Eulalia Valdés Ribalta, entre ellos el ingenio Santa Marta. ${ }^{45}$ De hecho, desde 1871, Tomás Ribalta pasó a recibir cómodamente en Barcelona los beneficios de tres haciendas diferentes, ubicadas todas en la región central de Cuba: los ingenios Santa Teresa, Santo Tomás y Santa Marta.

Poco podían imaginar en Cuba el menorquín Taltavull y el vasco Goytisolo que dos de sus vástagos, naturales ambos de Cienfuegos, acabarían contrayendo matrimonio en Barcelona. Y es que ambos hacendados

45 AHPVC, Registro de la Propiedad de Santa Clara, tomo III, folios 76-90; Protocolos de

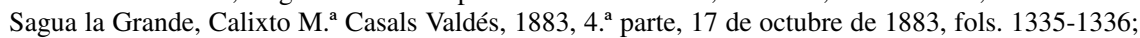


abandonaron Cuba para avecindarse en la capital catalana. El primero en hacerlo fue José P. Taltavull García. Junto a su hermano uterino Pedro A. Font García modificó la razón social de su casa de comercio cienfueguera (antes Font y Cía) para reconvertirla, en mayo de 1861, en García Taltavull y Cía. Ambos hermanastros adoptaron entonces el carácter de socios comanditarios preparando así su ulterior regreso a Cataluña. En febrero de 1864 se produjo una nueva contrata social. En aquella fecha, José P. Taltavull vivía ya en Barcelona, donde acabaría desarrollando una fecunda actividad empresarial (socio de la firma tabaquera Ros Aran y Cía, desde 1866; del Crédito Mutuo Fabril y Mercantil, desde 1873; y del Banco Hispano Colonial, desde 1876, entre otras compañías). Para sus inversiones, Taltavull contó con el flujo regular que le proporcionaban las ganancias de su ingenio cienfueguero pues, como se recogió en 1864, "los productos del ingenio Caridad se remitirán todos los años [desde Cuba] a España a disposición de D. José P. Taltavull". ${ }^{46}$

Agustín Goytisolo, por su parte, zarpó de Cuba con destino a la península en abril de 1870. Dejó sus intereses en la isla al cargo de su único yerno, Miguel Plana, y de su primogénito A. Fabián Goytisolo, y se instaló en Barcelona con su otro hijo varón y con sus cinco hijas. Desde la capital catalana, el veterano Goytisolo consiguió trasladar, en buena medida, su fortuna de Cienfuegos a Europa. Entre 1868 y 1882, los Goytisolo fueron capaces de transferir un total de dos millones de pesetas de Cuba a Cataluña. ${ }^{47}$ Deseoso como estaba de trasladar todo su capital de Cienfuegos a Barcelona, Agustín Goytisolo acostumbraba a compararse con Tomás Ribalta, sugiriendo constantemente a su hijo Fabián que emulase a sus administradores. En marzo de 1880, por ejemplo, le decía: "he sabido que también a Ribalta este año algo le obra, pues de los primeros 400 bocoyes le han mandado 25.000" pesos; dos meses más tarde, en mayo, Goytisolo insistía: "te empeñas en demostrarme que a Ribalta no le va también (sic) como supongo, pero lo cierto es que todos los años le mandan sumas que a veces son muy respetables sin que todo sea rentas de sus casas y negros, y también es cierto que no tengo yo allí un capital tan inferior a él para que nunca se me mande sino palabras y proyectos ... Ribalta recibió de los primeros azúcares 25.000 [pesos], no sé si habrá recibido

46 ANC, Protocolos Notariales, Gabriel Ramírez O’Brien, 1864, tomo I, fol. 107 y ss, 9 de febrero de 1864 .

47 Rodrigo, Martín: "Los Goytisolo. De hacendados en Cienfuegos a inversores en Barcelona", Revista de Historia Industrial, núm. 23, 2003, págs. 11-37. 
hasta ahora más pero puede contar con 50 ó 60 mil [pesos] más. Esos son los resultados que se apetecen, que lo demás no trae en esa [isla de Cuba] más que desengaños". ${ }^{48}$

En el otoño de 1882 se encontraron en una calle de Cienfuegos el comerciante Juan del Campo y el hacendado A. Fabián Goytisolo Digat (primogénito y apoderado de Agustín Goytisolo). Al poco, este último quiso relatar a su hermano Antonio, residente entonces en la capital catalana, la conversación recién mantenida por ambos diciéndole "que hablando con Dn Juan del Campo este Sr. me ha repetido ya por tres o cuatro ocasiones que Dn Tomás Ribalta había recibido [en Barcelona] de [su apoderado y sobrino político] Dn Juan de Oña un millón ochocientos mil pesos en oro. Yo supongo que desde que está en España y contando con el medio millón que heredó Dn. Tomás de Dn Pablo". ${ }^{49}$ Un millón ochocientos mil pesos en oro (o, lo que es lo mismo, nueve millones de pesetas) parece una cantidad realmente respetable. ¿Era el tal Juan de Oña un exagerado? En absoluto, en todo caso se había quedado corto. Unos meses antes de dicha conversación, en marzo de 1882, los apoderados de Tomás Ribalta habían protocolizado un inventario de sus bienes, obligados por la muerte de quien fuera su legítima esposa, Angela de León, acaecida en Sagua la Grande en octubre de 1880. Según dicho inventario, el patrimonio de la sociedad conyugal Tomás Ribalta-Ángela de León se evaluó entonces en 15.806 .830 pesetas, de los que 12.419.165 pesetas radicaban en Cataluña. Para conocer el monto total de su fortuna, a esa cifra deberíamos sumarle el valor de los bienes heredados de su hermano Pablo Luis Ribalta, que no aparecen en el inventario de la sociedad conyugal.

Tomás Ribalta Serra falleció en Barcelona en abril de 1887. Una vez descontada la porción que éste había pagado tras la muerte de su legítima esposa a sus hijastras (y a la vez sobrinas, dado que eran hijas del primer matrimonio de Ángela de León con el difunto José Ribalta Serra) ¿a cuanto ascendía su capital? En otras palabras, ¿qué fortuna dejó al morir Tomás Ribalta? Al hacerse eco de su muerte, La Vanguardia afirmaba que "don Tomás Ribalta ... poseía una de las primeras fortunas de España" mientras que al dar noticia del fallecimiento del "acaudalado capitalista D. Tomás Ribalta", un periodista del Diario de Barcelona decía que "según nos ha

48 FG, FAGL, caja 95, exp. 1, cartas de Agustín Goytisolo a su hijo A. Fabián, de 16 de marzo de 1880 y de 5 de mayo de 1880 .

49 FG, FAGL, caja 107, exp. 15, carta de A. Fabián Goytisolo a su hermano Antonio, de 9 de noviembre de 1882 . 
dicho persona que puede estar bien enterada, se calcula en nueve millones de duros la fortuna que ha dejado el señor Ribalta". ${ }^{50}$ En realidad, no eran nueve sino, casi, tres millones de duros (14.648.018 pesetas) el monto total de su fortuna, si le sumamos los valores que "por su larga comunidad de vida con Don Tomás Ribalta", su concubina, la joven Dolores Alquíza decaraba "haber recibido de él, en demostración de afecto y agradecimiento", unos títulos cuyo valor nominal era de 2.027.500 pesetas, pero que se cotizaban al fallecimiento del donante en 1.356 .977 pesetas. ${ }^{51}$ Una fortuna cimentada totalmente en el cultivo de la caña en sus ingenios cubanos. Con el tiempo, hasta cuatro de sus sobrinos-nietos cubanos abandonaron la isla para residir en Barcelona, disfrutando en la capital catalana de los beneficios, presentes y pasados, obtenidos merced a la explotación de los ingenios familiares, no sólo del Santa Teresa o el Santo Tomás, sino también de los ingenios Armonía, Manuelita y Purrio (propiedad de Juan de Oña Urría) o del ingenio Marina (propiedad de Francisco Santos de la Madrid), ambos sobrinos políticos (e hijastros) de Tomás Ribalta. ${ }^{52}$ De hecho, siguiendo la senda de su tío abuelo Tomás Ribalta tanto Concepción y Cecilia de Oña Ribalta como Eduardo y Tomás Santos de Lamadrid Ribalta pasaron a residir a Barcelona. Este último contribuiría a impulsar la Casa de América, una institución fundada en la capital catalana en 1911 con una participación importante de españoles nacidos en el nuevo continente. ${ }^{53}$

Los patrimonios acumulados en vida por Agustín Goytisolo y por José P. Taltavull, cimentados en el azúcar cubano, aun siendo inferiores al de Tomás Ribalta, fueron igualmente notables. A la muerte de José P. Taltavull, en noviembre de 1881, sus albaceas calcularon en 3.478.985 pesetas la fortuna neta que Taltavull había conseguido transferir a Cataluña (incluyendo la que heredó de sus otros familiares indianos) en los últimos años de su vida. Al finalizar dicho inventario, sin embargo, sus propios albaceas declararon que "a más de los bienes existentes en la península forman parte de la herencia [de José P. Taltavull] otros en Ultramar" cuyo ava-

50 Diario de Barcelona, 14 de abril de 1887-tarde, págs. 4403-4404; La Vanguardia, 14 de abril de 1887, pág. 2308, que añadía que Ribalta "disfrutaba una renta de 2.000 duros diarios".

51 AHPB, Miguel Martí Sagristá, 1887, 1. a parte, 25 de abril de 1887, fol.s 857-879; 29 de abril de 1887, fols. 892-897; . $^{\text {a }}$ parte, 10 de mayo de 1887, fols. 962-964; 15 de julio de 1887, fols. 1495 y ss; 3. a parte, fols. 1727-1728, 24 de septiembre de 1887; AHPVC, Protocolos de Sagua la Grande, Calixto M. ${ }^{\text {a }}$ Casals Valdés, 1888,1 . $^{\mathrm{a}}$ parte, 26 de enero de 1888 , fols. 104-180.

52 AHPVC, Protocolos de Sagua la Grande, Calixto M. ${ }^{a}$ Casals Valdes, 1890, 11 de enero de 1890, fols. 15-45.

53 Dalla Corte, Gabriela: Casa de América en Barcelona, 1911-1947, Madrid, LID, 2005, págs. 108-109. 
luo no pudieron recoger entonces y entre los que se mantenía el ingenio Caridad. ${ }^{54}$ Cinco años después, en 1886, falleció Agustín Goytisolo Lezarzaburu, también en Barcelona. El caudal líquido de la porción de su fortuna radicada en Barcelona, sumó 3.117.406 pesetas, cifra que tampoco incluye el avaluo de sus bienes e ingenios cubanos. ${ }^{55}$ Sabemos, por otro lado, que Francisco de Sola se había trasladado a la península meses antes que el propio Agustín Goytisolo. Desde su domicilio en la capital catalana, Sola siguió recibiendo en su vejez los beneficios de las zafras de su ingenio Cieneguita, gestionado por sus hijos.

Por último, cabe señalar que hubo otros hombres de negocio cienfuegueros, enriquecidos también merced al azúcar, que acabaron trasladando su residencia a Cataluña. Ese fue el caso de Agustín Irizar de Clouet, sobrino del que fuera fundador en 1827 de la colonia Fernandina de Jagua, primer nombre de Cienfuegos, Leopoldo de Clouet. El tal Agustín Irizar se enriqueció a la sombra del hombre más rico de Cienfuegos y, al decir de Roland T. Ely, más rico de toda Cuba, el hacendado Tomás Terry, propietario de numerosas fincas cañeras. Uno de sus ingenios, el Caracas, comprado por Terry en 1862, estaba situado precisamente en las cercanías del San Agustín, propiedad de los Goytisolo. Irizar empezó a trabajar para Terry al menos desde 1851: en esa fecha tenía asignado un sueldo de 2.000 pesos al año. Partidario de remunerar bien a sus empleados, Terry fue subiendo progresivamente la retribución asignada a Irizar: en 1859 su sueldo alcanzaba ya los 9.000 pesos anuales que subieron a 12.000 pesos a partir de 1864. Como ha escrito Roland T. Ely, Tomás Terry "a fines de 1866 ofreció a su empleado principal, Agustín Yrizar, una participación en el negocio. Yrizar, que desde hacía tiempo estaba autorizado para firmar en nombre de Terry por poder ... naturalmente aceptó la proposición que se le formulaba. Aparte del prestigio que connotaba el ser socio de un multimillonario, recibiría el $10 \%$ de las ganancias netas de la firma". El propio Ely ha destacado como la participación de Irizar en los beneficios de Terry marcó un hito en el incremento patrimonial del primero quien, en 1867, recibió de esa forma 36.727 pesos oro (equivalentes por lo menos a unos 45.000 dólares de entonces), triplicando su anterior asignación. Para el resto de años Ely no ofrece datos precisos de los ingresos de Agustín Irizar pero podemos suponer que fueron notables. Y más a partir de 1877, fecha

54 AHPB, José Jordana, 1882, 1. ${ }^{a}$ parte, 16 de febrero 1882, fols. 177-238; 13 de mayo de 1882, fols. 575-577; 23 de mayo de 1882, fols. 629-631.

55 AHPB, José María Vives Mendoza, 1887, 1. a parte, 18 de marzo de 1887, fols. 662-692. 
en que su participación en las ganancias de la firma de Tomás Terry y Cía subió hasta alcanzar el 20 por 100 del total. De hecho, el capital de Tomás Terry subió de 3.090 .593 pesos en 1860 , a 7.891 .784 pesos diez años más tarde y a 13.763.594 pesos en 1880. A finales de ese último año, es decir, más de tres décadas después de empezar a trabajar con el creso cubano, Agustín Irizar se retiró de la sociedad Tomás Terry y Cía, abandonando poco después la isla y pasando a residir a Barcelona, donde fallecería años después. ${ }^{56}$ En la capital catalana pudo vivir precisamente de las rentas producidas por su vinculación con el dulce cubano.

Como también lo pudieron hacer los catalanes José Carbó y Joaquín Fábregas, aunque este último por poco tiempo. Fábregas zarpó de Cuba prácticamente a la par que Agustín Irizar, en 1881. Se trasladó directamente a su residencia en el barcelonés Paseo de Gracia. Allí murió meses después, en febrero de 1882, soltero y sin hijos. En consecuencia, fueron sus tres hermanos y los hijos de sus tres hermanas difuntas, quienes se repartieron el capital del indiano fallecido, tal como éste había expresado en sus últimas voluntades. Unos herederos que vivían todos ellos en Cataluña (en Piera, Esparreguera, Barcelona o Sant Pere de Terrassa), de manera que, también en este caso, el conjunto del caudal acumulado por la actividad empresarial del médico y hacendado Joaquín Fábregas en Sagua la Grande revirtió entera y totalmente en el Principado. ${ }^{57} \mathrm{El}$ también hacendado José Carbó Martinell, por su parte, había regresado a Barcelona en 1867, dejando a su primogénito al frente del ingenio Santa Catalina, en Yaguajay. Destinó parte de su capital a la compra de solares e inmuebles en la capital catalana. De hecho, entre septiembre de 1869 y febrero de 1872 José Carbó invirtió, al menos, 948.620 pesetas en dicha actividad. ${ }^{58}$

56 Ely, R. T.: Cuando reinaba ... Las referencias a Agustín Irizar en las páginas 392-393; los datos de la fortuna de Tomás Terry en la pág. 406. La transformación de Tomás Terry y Cía en AHPC, Emigdio Nieto Guiote, Manual de 1881, 19 de abril de 1881, fols. 549-552. Según A. Fabián Goytisolo, Agustín Irizar zarpó de Nueva York con destino a Liverpool en agosto de 1881; cfr. FG, FAGL, caja 96, exp. 10: carta de A. Fabián Goytisolo a su padre, de 15 de agosto de 1881. Llegó después a Barcelona donde residía en 1896, cfr. AHPB, José María Vives Mendoza, 1896, 3. a parte, 14 de julio de 1896, fol. 2606 y ss.

57 AHPB, Mariano Barallat, 1882, 14 de mayo de 1882, fols. 232-235.

58 Tras la muerte de José Carbó Martinell, le correspondió a su hijo homónimo, José Carbó Cantó, finalizar las obras de construcción de diferentes inmuebles en los número 7 y 9 de la calle Pelayo y en el número 13 de la calle Balmes, por los que pagó 185.440 pesetas más: AHPB, Magín Soler Gelada, 1867, 3. ${ }^{\text {a }}$ parte, 8 de noviembre de 1867, fols. 1476-1480; 1869, 1 . $^{\text {a }}$ parte, 20 de marzo de 1869, fols. 421-425; José Ramón Pasqués, 1868, 30 de marzo de 1868, fols. 111-116; Miguel Martí Sagristá, 1870, 1. a parte, 21 de mayo de 1870, fols. 541-542; 1877, 2. ${ }^{\text {a }}$ parte, 30 de mayo de 1877 , fols. 1033-1058. 


\section{Conclusiones}

Podría ampliar este análisis microhistórico a partir de la inclusión de diversos hombres de negocio quienes, desde Cataluña, seguían manteniendo la propiedad de sus ingenios en Cuba. Por ejemplo, el banquero y político de Vilanova i la Geltrú, Francisco Gumá Ferran, era propietario de los ingenios Santa Sofía, en Matanzas, y Angelita, en Güines. Nemesio Ronsart Sans, por su parte, era propietario de la finca cañera El Placer, en Victoria de las Tunas. Encontramos, por otro lado, diferentes ingenios en la región oriental de Cuba cuyos propietarios residían en Barcelona. Ese fue el caso de Fernando Perfecto Robert, propietario del ingenio Manacal, en Santiago de Cuba. También el cántabro Antonio López y López mantuvo la propiedad de las haciendas Santa Ana, Soledad y Carmen tras su instalación en Barcelona, en 1855. No las vendió, de hecho, hasta siete años después, en $1862 .{ }^{59}$

Una tía de su mujer, la dominicana Cecilia Coulange, viuda del catalán Magín Massó, heredó de su difunto marido la mitad del ingenio Abundancia, sito en la región de Santiago de Cuba, cuya propiedad vendió en noviembre de 1857 a su hijo, Magín Masó Coulange, y a su yerno, el toledano Benito Rubio López de Bocanegra, al poco de trasladar su residencia a la capital catalana. Este último seguiría los pasos de su suegra, avecindándose asimismo a Barcelona. Además, Rubio mandó construir una lujosa mansión en Madrid, consiguiendo fondos mediante la hipoteca no sólo de su interés en el ingenio Abundancia, sino también de sus otros dos ingenios, nombrados Laguna y San Andrés, ubicados ambos en las cercanías de Santiago de Cuba. ${ }^{60}$ Por su parte, el Auditor retirado de Marina, el cubano José Ramón de Villalón, había decidido avecindarse asimismo en Barcelona. Durante unos años, antes de que los mambises quemaran sus propiedades en la región santiaguera, Villalón fue recibiendo comodamente las ganancias producidas por sus dos cafetales así como por "un ingenio de fabricar azúcar que allí existió e incendiaron los insurrectos nombrado Santa Cruz del Juncal, en el cuartón de Maroto, partido de Jutinicú”, cuya

59 AHPSC, Escribanía de Giró, protocolo 283, fol. 10 y ss; Escribanía Real de Manuel Caminero, protocolo 112, fol. 216 y ss; AHPB, Ramón de Miquelerena, 1863, 1. ${ }^{\text {a }}$ parte, 2 de junio de 1863.

60 AHPSC, Escribanía de Juan Giró, 1857, 9-XI-1857, fols. 644 y ss; AHPB, Jaime Rigalt, 1857, 3 de julio de 1857, fols. 403-407; Ramón de Miquelerena, 1859, 1. a parte, 14 de marzo de 1859, fol. 124 y ss; 1861,1 . $^{\text {a }}$ parte, 13 de febrero de $1861 ; 2{ }^{\text {a }}$ parte, 3 de octubre de 1861 , fol. 179 y ss. 
mitad le pertenecía. Sus socios en el negocio, es decir, los propietarios de la otra mitad de la finca cañera, habían sido "los hermanos Don Eligio y Don Urbano Ros, residentes tambien hoy [escribía Villalón en 1872] en esta capital". ${ }^{61}$ Incluso podría realizarse una aproximación similar tomando diferentes zonas de Puerto Rico. De hecho, tanto José Milá de la Roca como su yerno Gerónimo Rabassa (propietarios de la hacienda Cañoverde, en Ponce) así como Sebastián Plaja Vidal (propietario del ingenio Vista Alegre, también en Ponce) o como Juan Anglada Carreras (propietario de una finca cañera en Jabucoa denominada simplemente Ingenio), acabaron los últimos años de su vida en Barcelona, disfrutando de las ganancias producidas por sus haciendas puertorriqueñas.

De cualquier forma, considero que con el análisis de las trayectorias vitales de los hermanos Ribalta Serra, José P. Taltavull, Agustín Goytisolo, Francisco de Sola, Agustín Irizar, Joaquín Fábregas y José Carbó es suficiente para apreciar una realidad que, por supuesto, fue más allá de los casos estudiados. Un número importante de los hacendados cubanos acabaron viviendo fuera de la isla. Para el caso español, el trabajo de Ángel Bahamonde y José Cayuela ha dado cuenta de muchos de ellos. ${ }^{62}$ El fenómeno, por supuesto, no se limitaba a un eje bilateral Cuba-España: Gran Bretaña, Francia y los Estados Unidos fueron asimismo territorios de llegada para numerosos hacendados enriquecidos en Cuba. Además, algunos importadores norteamericanos de azúcar cubano acabaron fomentando o comprando ingenios en Cuba. En Sagua la Grande, por ejemplo, encontramos a Augustus Hemenway (de Boston), dueño del ingenio San Jorge; o los Howland (de Nueva York), propietarios de la hacienda El Dorado. Ese sería el caso, por ejemplo, de los Atkins, importadores de azúcar cubano radicados en Boston quienes acabarían comprando fincas cañeras en las cercanías de Cienfuegos. En un trabajo de corte autobiográfico Edwin F. Atkins da noticias de diferentes comerciantes de azúcar de Boston quienes asimismo se interesaron directamente por la producción del dulce en Cuba, tales como Benjamin Burgess \& Sons. ${ }^{63}$ La extensión del fenómeno, en fin, resta aun por ser conocida y estudiada en toda su complejidad y profundidad.

No obstante, las evidencias acumuladas (y aquí se han presentado, en detalle, algunas) son suficientes para apuntar algunas conclusiones. Tanto hacendados de Cuba como exportadores cubanos de azúcar (o importado-

61 AHPB, Fernando Ferran, 1872, 1. a parte, 1 de febrero de 1872, fols. 242-248.

62 Bahamonde, Ángel; Cayuela, José: Hacer las Américas, Madrid, Alianza, 1992.

63 Atkins, Edwin F.: Sixty years in Cuba, New York, Arno Press, 1980. 
res de dulce radicados en los Estados Unidos) optaron por situar sus capitales fuera de la isla. Unos capitales producidos en buena medida por el azúcar. Parece claro que mientras que la expansión de la caña de azúcar en la isla se tradujo en la deforestación de su cubierta forestal; es decir, mientras que la expansión de la caña significó una pesada herencia en términos de pérdida de masa forestal y de pérdida de biodiversidad, sobre la que no existe posibilidad de marcha atrás, los beneficios del proceso emigraron, en buena medida, a otras latitudes. Fueron estas regiones, europeas y norteamericanas (y aquí hemos analizado particularmente la relación Las VillasBarcelona) las que acabaron captando las ganancias del azúcar. O, si se prefiere, los beneficios del dulce. En el caso que nos ocupa, puede incluso establecerse una relación directa entre el capital transferido desde Cuba y su contribución al crecimiento económico del único territorio español realmente industrializado en el tercer cuarto del siglo XIX. Parece claro, pues, que Cataluña contrajo en los años de la deforestación de la isla una deuda ecológica con Cuba.

Recibido el 6 de octubre de 2005 Aceptado el 12 de febrero de 2006 